\title{
Higher-Order Hybrid Method for Curl-Conforming Elements on Tetrahedrons and Bricks
}

\author{
J. Winges*
}

\begin{abstract}
We present a brick-tetrahedron hybrid finite-element method with higher-order basis functions for solving electromagnetic field problems. For the brick-shaped elements, we use mass lumping for interpolatory basis-functions of incomplete order, which yields a diagonal mass-matrix. Hierarchical basis functions of incomplete and/or complete order are used for the tetrahedral elements. We enforce tangential continuity at the brick-tetrahedron interface in the weak sense. Our hybrid method correctly reproduces the lowest eigenvalues with correct multiplicity for a closed cavity-resonator. For tests in a rectangular waveguide, we demonstrate that the hybrid interface yields a low reflection coefficient.
\end{abstract}

\section{INTRODUCTION}

Structured grids are popular for Maxwell's equations since they are easy to use and allow for the formulation of computationally efficient methods such as the finite-difference time-domain scheme [1]. In the context of the finite-element method (FEM), structured meshes of brick-shaped elements make it possible to achieve so-called mass lumping [2]. The mass lumping yields a diagonal mass-matrix, which makes it feasible to use explicit time-stepping schemes. In addition, the FEM works well with body-conforming unstructured meshes of tetrahedrons, which are suitable for complex geometry and local mesh-refinement. Moreover, the FEM on the tetrahedrons can be formulated in terms of hierarchical basis functions [3], which yields considerable flexibility as the polynomial order of the basis functions can be varied from element to element. However, tetrahedral elements are unsuitable for mass lumping and, as a consequence, time-domain formulations on unstructured meshes require a matrix inversion for each time step.

Thus, a computationally attractive solution is to combine (i) structured meshes of bricks in large homogeneous regions with (ii) unstructured meshes of tetrahedrons only in regions where it is required due to complex geometry or rapid variations in the field solution. We represent the electric field by means of curl-conforming elements [4], which are well-suited for solving Maxwell's equations. At the interface between the brick-shaped and tetrahedral elements,

\footnotetext{
${ }^{*}$ Department of Signals and systems, Chalmers University of Technology, Göteborg, Sweden; e-mail: \{winges, rylander\}@chalmers.se
}

\author{
T. Rylander*
}

the degrees of freedom associated with the two different element types must be related to each other in an appropriate manner. Early attempts include various interpolation approaches across the hybridinterface $[5,6]$, which often yield non-symmetric discrete operators that fail to preserve the reciprocity of Maxwell's equations. It is possible to construct stable hybrid methods of the lowest order, where the reciprocity of Maxwell's equations is preserved. One such option is to use a layer of pyramidal elements in-between the brick-shaped and tetrahedral elements [7] in order to formulate a FEM that is curl-conforming throughout the entire hybrid mesh. However, the pyramids makes programming and mesh generation more difficult. Moreover, it is not trivial to extend such an approach to higher-order elements.

In this article, we consider a hybrid method with higher-order basis functions that connect the tetrahedral mesh directly to the brick-shaped mesh. For the brick-shaped elements, we use basis functions [2] that belong to an incomplete polynomialsubspace of $\mathcal{H}(\operatorname{curl}, \Omega)$ of order $p^{\mathrm{b}}$, where these basis functions allow for mass lumping. For the tetrahedral elements, we use hierarchical basisfunctions [3] of either incomplete or complete polynomial order $p^{\mathrm{t}}$. Consequently, this construction allows for a discontinuous tangential component of the electric field across the brick-tetrahedron interface. For the lowest-order hybrid, it is possible to establish conditions at the interface that preserve the null-space of the curl-curl operator despite that the electric field features a discontinuous tangential component [8]. In this article, we achieve tangential continuity across the interface in the weak sense [9]. This construction has similarities with discontinuous Galerkin (DG) methods [10, 11], where a penalty term is used to weakly enforce tangential continuity across the element faces for the entire mesh.

\section{HYBRID METHOD}

We divide the computational domain $\Omega$ in two parts: (i) $\Omega^{\mathrm{t}}$ is discretized by tetrahedral elements, and (ii) $\Omega^{\mathrm{b}}$ is discretized by brick-shaped elements. The hybrid interface is denoted $\Gamma^{\mathrm{I}}=\partial \Omega^{\mathrm{t}} \cap \partial \Omega^{\mathrm{b}}$. Here, the discretization is constructed such that the 
rectangular face of a brick, which coincides with $\Gamma^{\mathrm{I}}$, is divided on the diagonal into two triangular faces associated with the tetrahedrons of the unstructured mesh. Further, we let $\hat{\boldsymbol{n}}$ be the unit normal vector associated with $\Gamma^{\mathrm{I}}$ that points away from $\Omega^{\mathrm{b}}$.

\subsection{The model problem}

For a source free region $\Omega$ with homogeneous material parameters, Maxwell's equations for a timeharmonic electric field $\boldsymbol{E}(\boldsymbol{r}, \omega)$ yields

$$
\begin{array}{rlrl}
\nabla \times \nabla \times \boldsymbol{E}-\omega^{2} \mu_{0} \epsilon \boldsymbol{E}=\mathbf{0} & & \text { in } \Omega^{\mathrm{t}} \text { and } \Omega^{\mathrm{b}}, \\
\hat{\boldsymbol{n}} \times \llbracket \boldsymbol{E} \rrbracket=\mathbf{0} & & \text { on } \Gamma^{\mathrm{I}}, \\
\hat{\boldsymbol{n}} \times \llbracket \nabla \times \boldsymbol{E} \rrbracket & =\mathbf{0} & & \text { on } \Gamma^{\mathrm{I}}, \\
\hat{\boldsymbol{n}} \times \boldsymbol{E} & =\mathbf{0} & & \text { on } \Gamma=\partial \Omega,
\end{array}
$$

where $\llbracket \boldsymbol{E} \rrbracket=\boldsymbol{E}^{\mathrm{b}}-\boldsymbol{E}^{\mathrm{t}}$ is the jump of $\boldsymbol{E}$ at the interface $\Gamma^{\mathrm{I}}$. Here, the outer boundary $\Gamma=\partial \Omega$ of the domain $\Omega$ is a closed perfect electric conductor (PEC) surface.

\section{$2.2 \quad$ FEM formulation}

We expand the electric field in terms of curlconforming basis functions and apply Galerkin's method, where continuity of the tangential component is enforced in the weak sense [9] at the interface $\Gamma^{\mathrm{I}}$. This yields the discrete eigenvalue problem

$$
\left(\mathbf{K}-\mathbf{K}^{\mathrm{I}}+\gamma \mathbf{K}^{\mathrm{I}_{0}}\right) \mathbf{e}=\epsilon_{0} \mu_{0} \omega^{2} \mathbf{M e},
$$

where $\mathbf{e}$ is the solution vector that represent the electric field. Further, we have the matrices

$$
\begin{aligned}
K_{i, j} & =\left(\nabla \times \boldsymbol{w}_{i}^{\mathrm{t}}, \nabla \times \boldsymbol{w}_{j}^{\mathrm{t}}\right)_{\Omega^{\mathrm{t}}} \\
& +\left(\nabla \times \boldsymbol{w}_{i}^{\mathrm{b}}, \nabla \times \boldsymbol{w}_{j}^{\mathrm{b}}\right)_{\Omega^{\mathrm{b}}}, \\
K_{i, j}^{\mathrm{I}} & =\left(\hat{\boldsymbol{n}} \times \llbracket \boldsymbol{w}_{i} \rrbracket,\left\{\left\{\nabla \times \boldsymbol{w}_{j}\right\}\right\}_{\alpha}\right)_{\Gamma^{\mathrm{I}}} \\
& +\left(\left\{\left\{\nabla \times \boldsymbol{w}_{i}\right\}_{\alpha}, \hat{\boldsymbol{n}} \times \llbracket \boldsymbol{w}_{j} \rrbracket\right)_{\Gamma^{\mathrm{I}}},\right. \\
K_{i, j}^{\mathrm{I}_{0}} & =\left(\hat{\boldsymbol{n}} \times \llbracket \boldsymbol{w}_{i} \rrbracket, h^{-1} \hat{\boldsymbol{n}} \times \llbracket \boldsymbol{w}_{j} \rrbracket\right)_{\Gamma^{\mathrm{I}}}, \\
M_{i, j} & =\left(\boldsymbol{w}_{i}^{\mathrm{t}}, \epsilon_{\mathrm{r}} \boldsymbol{w}_{j}^{\mathrm{t}}\right)_{\Omega^{\mathrm{t}}}+\left(\boldsymbol{w}_{i}^{\mathrm{b}}, \epsilon_{\mathrm{r}} \boldsymbol{w}_{j}^{\mathrm{b}}\right)_{\Omega^{\mathrm{b}}} .
\end{aligned}
$$

where $(\boldsymbol{a}, \boldsymbol{b})_{\Omega}=\int_{\Omega} \boldsymbol{a} \cdot \boldsymbol{b} d \Omega$ denotes the inner product in the volume $\Omega$ and $(\boldsymbol{a}, \boldsymbol{b})_{\Gamma}=\int_{\Gamma} \boldsymbol{a} \cdot \boldsymbol{b} d \Gamma$ on the surface $\Gamma$. Further, $\{\{\boldsymbol{E}\}\}_{\alpha}=\alpha \boldsymbol{E}^{\mathrm{b}}+(1-\alpha) \boldsymbol{E}^{\mathrm{t}}$ is the average electric field at the interface and $h$ denotes the local element size. The value of $\alpha \in[0,1]$ does not influence the solution [9] and, for simplicity, we use $\alpha=1$ below.

The matrices $\mathbf{K}$ and $\mathbf{M}$ are block diagonal, where each block consists of the regular stiffness and mass matrices for the respective element types. $\mathbf{K}^{\mathrm{I}}$ and $\mathbf{K}^{\mathrm{I}_{0}}$ are constructed from integration of the jump and average terms over the hybrid interface.
In a regular curl-conforming $\mathrm{FE}$ formulation (e.g. using only one element type), the stiffness matrix is positive semi-definite and the electrostatic field solutions $\boldsymbol{E}=-\nabla \phi$ spans its null-space.

\section{RESULTS}

\subsection{Resonances in a metal cavity}

We compute the resonant frequencies for a cavity resonator with PEC walls, where the cavity is shaped as a cube of side $1 \mathrm{~m}$. An example of a hybrid mesh is shown in Fig. 1, where we combine cube-shaped elements with tetrahedrons.

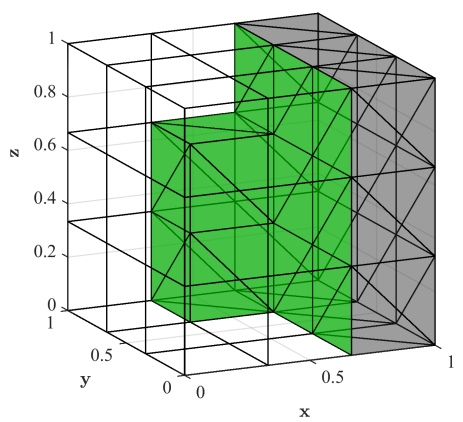

Figure 1: Hybrid mesh of a cubic cavity. The cubeshaped elements are transparent whereas the tetrahedral elements are shown in gray and the hybrid interface is shown in green.

The generalized eigenvalue problem in Eq. (2) is solved for the stabilization parameter $\gamma=10^{3}$, where we in all test cases that follow use incomplete order for the basis functions on the cube-shaped elements. The eigenfrequencies are shown in Fig. 2 for hybrid meshes with linear $(p=1)$ and quadratic basis functions $(p=2)$. The crosses show the results for the hybrid by Degerfeldt and Rylander [8], which features the lowest-order elements for both the cubes and the tetrahedrons with a strong coupling of the degrees of freedom at the interface. We denote this approximation by $p=1_{\mathrm{i} \text {,strong, where }}$ the subindex i corresponds to incomplete-order elements on the tetrahedrons. This yields a discontinuous electric field at the interface. However, the tangential electric field is continuous for any constant field and the null space of the curl-curl operator is preserved. (We find that imposing continuity in the weak sense for the lowest-order elements, i.e. $p=1_{\mathrm{i} \text {,weak }}$, fails to converge towards the correct spectrum, regardless of the value of $\gamma$.)

Linear complete-order basis functions on the tetrahedrons yields a tangential electric field at the interface that can express any linear variation in the field and, thus, this option allows for a perfect fit of the tangential electric field to the (incomplete) lowest-order basis functions on the cube-shaped el- 
ements. For this case, we use the weak coupling at the interface and denote the result by $p=1_{\mathrm{c} \text {,weak }}$, where $\mathrm{c}$ denotes complete-order basis functions on the tetrahedrons.

We also show the spectrum for tangential continuity imposed in the weak sense when quadratic basis-functions are used for the hybrid mesh: $p=$ $2_{\mathrm{i} \text {,weak }}$ - incomplete-order basis on the tetrahedrons; and $p=2_{\text {c,weak }}$ - complete-order basis on the tetrahedrons. It should be emphasized that the electric field is represented by incomplete-order quadratic basis-functions on the cube-shaped elements. Thus, $p=2_{\mathrm{i} \text {, weak }}$ yields a discontinuous electric field at the interface but, for an appropriate choice of the stabilization parameter $\gamma$, the null space of the curl-curl operator is preserved by Eq. (2) and the lowest non-zero eigenvalues converge towards the analytical result.

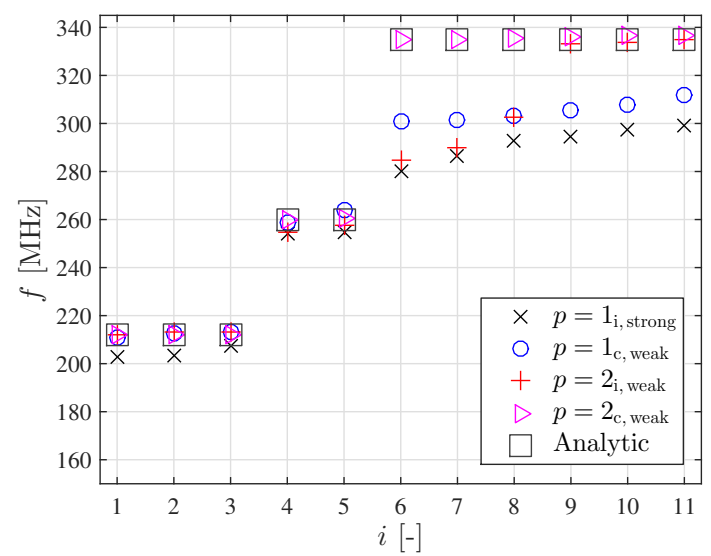

Figure 2: Eigenfrequencies for a PEC cavity shaped as a cube. Subscript i and c refers to incomplete- and complete-order basis functions on the tetrahedrons, respectively. Results are shown for strong coupling of the degrees of freedom [8] for the lowest-order basis functions and tangential continuity imposed in the weak sense for higher-order basis functions.

Figure 3 shows the relative error $\left|f-f_{\mathrm{a}}\right| / f_{\mathrm{a}}$ as a function of the number of degrees of freedom: (a) - incomplete-order basis on the tetrahedrons; and (b) - complete-order basis on the tetrahedrons. (Here, $f_{\mathrm{a}}$ denotes the analytical eigenfrequency.) The crosses indicate curves associated with the three lowest eigenfrequencies $(f=212 \mathrm{MHz})$ and circles the next group of two eigenfrequencies $(f=$ $260 \mathrm{MHz}$ ). We note that Eq. (2) yields a leading error that is approximately proportional to $h^{2}$ for the linear basis-functions and $h^{4}$ for the quadratic basis-functions.

\subsubsection{Reflection coefficient of interface}

Next, we consider a rectangular waveguide of width $1 \mathrm{~m}$ and height $0.5 \mathrm{~m}$, which is discretized by cube-

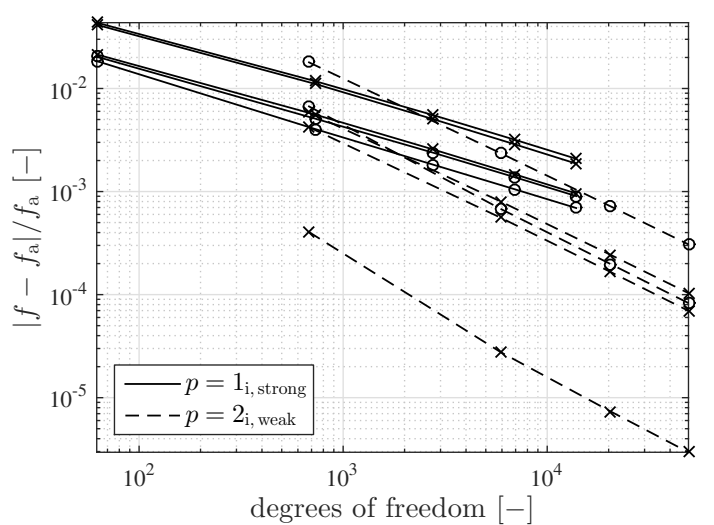

(a) Incomplete-order

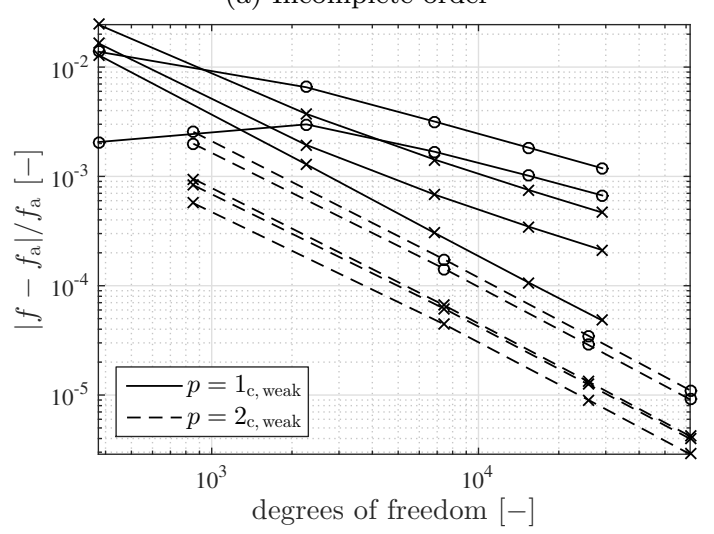

(b) Complete-order

Figure 3: Relative error in the resonance frequency of the five lowest resonance frequencies versus the number of degrees of freedom in the eigenvalue problem.

shaped elements of side $0.5 \mathrm{~m}$. Here, one layer (perpendicular to the cylinder axis of the waveguide) of cube-shaped elements is replaced by tetrahedrons. In this setting, we use a Robin boundary condition [12] at the waveguide ports at the two ends of the waveguide. Thus, we can launch the fundamental $\mathrm{TE}_{10}$ waveguide mode from one of the two ports and propagate it towards the layer of tetrahedrons. The reflected and transmitted field is extracted from the solution at the waveguide ports.

The average reflection coefficient for the hybrid method with linear and quadratic basis-functions of incomplete and complete order are presented in Tab. 1, where the average is computed for the frequency range $157 \mathrm{MHz}$ to $292 \mathrm{MHz}$. As a reference, we also present the reflection coefficient for a discretization with only cube-shaped elements, i.e. without the layer of tetrahedrons. This yields a non-zero reflection coefficient due to the port boundary-conditions that involve the analytic wavenumber. 


\begin{tabular}{|l|c|c|}
\hline Method & $p=1$ & $p=2$ \\
\hline Hybrid, incomplete & - & $-40.7 \mathrm{~dB}$ \\
Hybrid, complete & $-21.0 \mathrm{~dB}$ & $-50.6 \mathrm{~dB}$ \\
Reference, only cubes & $-24.6 \mathrm{~dB}$ & $-54.3 \mathrm{~dB}$ \\
\hline
\end{tabular}

Table 1: Average $\left|S_{1,1}\right|$ coefficients in $\mathrm{dB}$ found for a rectangular waveguide with a layer of tetrahedrons embedded in a structured mesh of cube-shaped elements.

\section{Conclusions}

We have presented a higher-order hybrid method using tetrahedrons and bricks for solving electromagnetic field problems. On the bricks, we use curl-conforming basis functions of incomplete order which allow for mass lumping and explicit timestepping. We use hierarchical curl-conforming basis functions of incomplete or complete order on the tetrahedrons. The tangential continuity across the hybrid interface is enforced in a weak sense.

The hybrid method employs a stabilization parameter which can be chosen. For an appropriate choice of the stabilization parameter, the hybrid method correctly reproduces the lowest non-zero eigenfrequencies for cavity resonators given that we use complete first-order or higher-order basis functions for the tetrahedrons. For a rectangular waveguide, the hybrid method achieves a low reflection coefficient from a single layer of tetrahedrons embedded in a structured mesh of cube-shaped elements.

\section{Acknowledgments}

This work was supported in part by the Swedish Research Council (dnr 2010-4627) in the project "Model-based Reconstruction and Classification Based on Near-Field Microwave Measurements". The computations were performed on resources at Chalmers Centre for Computational Science and Engineering (C3SE) provided by the Swedish National Infrastructure for Computing (SNIC).

\section{References}

[1] K. Yee, "Numerical solution of initial boundary value problems involving Maxwell's equations in isotropic media," IEEE Trans. Antennas Propag., vol. 14, pp. 302-307, 1966.

[2] G. C. Cohen, Higher-Order Numerical Methods for Transient Wave Equations. Springer, 2002.

[3] $\mathrm{P}$. Ingelström, "A new set of $\mathrm{H}$ (curl)conforming hierarchical basis functions for tetrahedral meshes," IEEE Trans. Microw. Theory Tech., vol. 54, pp. 106-114, 2006.

[4] J. C. Nédélec, "Mixed finite elements in R3," Numer. Math., vol. 1, no. 35, pp. 315-341, 1980.

[5] A. Monorchio and R. Mittra, "A hybrid finite-element finite-difference time-domain (FE/FDTD) technique for solving complex electromagnetic problems," IEEE Trans. Microw. Guid. Wave Lett., vol. 8, pp. 93-95, 1998.

[6] M. El Hachemi, O. Hassan, K. Morgan, D. Rowse, and N. Weatherill, "A low-order unstructured-mesh approach for computational electromagnetics in the time domain.," Philosph. trans. Ser. A, Math., Phys. eng. sci., vol. 362, pp. 445-69, Mar. 2004.

[7] T. Rylander and A. Bondeson, "Stable FEMFDTD hybrid method for Maxwell's equations," Comput. Phys. Commun., vol. 125, pp. 75-82, Mar. 2000.

[8] D. Degerfeldt and T. Rylander, "A bricktetrahedron finite-element interface with stable hybrid explicit-implicit time-stepping for Maxwell's equations," J. Comput. Phys., vol. 220, pp. 383-393, Dec. 2006.

[9] A. Hansbo, P. Hansbo, and M. G. Larson, "A finite element method on composite grids based on Nitsche's method," ESAIM Math. Model. Numer. Anal., vol. 37, pp. 495-514, 2003.

[10] P. Houston and I. Perugia, "Discontinuous Galerkin methods for the time-harmonic Maxwell equations," Numer. Math. Adv. Appl., pp. 483-492, 2004.

[11] T. Warburton and M. Embree, "The role of the penalty in the local discontinuous Galerkin method for Maxwell's eigenvalue problem," Comput. Methods Appl. Mech. Eng., vol. 195, pp. 3205-3223, May 2006.

[12] J. Jianming, The Finite Element Method in Electromagnetics. Wiley-IEEE Press, 2nd ed., 2002. 